\title{
Four-octyl itaconate activates Nrf2 cascade to protect osteoblasts from hydrogen peroxide- induced oxidative injury
}

Yuehuan Zheng ${ }^{1,2}$, Zhe Chen ${ }^{1}$, Chang She ${ }^{3}$, Yazhou Lin ${ }^{1}$, Yuan Hong ${ }^{1,2}$, Liqiang Shi ${ }^{1}$, Yingzi Zhang ${ }^{3}$, Peng Cao ${ }^{1}$ and Xiangyang $\mathrm{Xu}^{1,2}$

\begin{abstract}
Four-octyl itaconate (4-OI) is the cell-permeable derivative of itaconate that can activate Nrf2 signaling by alkylating Keap1's cysteine residues. Here, we tested the potential effect of 4-Ol on hydrogen peroxide $\left(\mathrm{H}_{2} \mathrm{O}_{2}\right)$-induced oxidative injury in osteoblasts. In OB-6 cells and primary murine osteoblasts, 4-OI was able to activate Nrf2 signaling cascade and cause Keap1-Nrf2 disassociation, Nrf2 protein stabilization, cytosol accumulation, and nuclear translocation. 4-Ol also augmented antioxidant-response element reporter activity and promoted expression of Nrf2-dependent genes ( $\mathrm{HO1}$, NQO1, and GCLC). Pretreatment with 4-Ol inhibited $\mathrm{H}_{2} \mathrm{O}_{2}$-induced reactive oxygen species production, cell death, and apoptosis in osteoblasts. Furthermore, 4-OI inhibited $\mathrm{H}_{2} \mathrm{O}_{2}$-induced programmed necrosis by suppressing mitochondrial depolarization, mitochondrial cyclophilin D-ANT1 (adenine nucleotide translocase 1)-p53 association, and cytosol lactate dehydrogenase release in osteoblasts. Ectopic overexpression of immunoresponsive gene 1 (IRG1) increased endogenous itaconate production and activated Nrf2 signaling cascade, thereby inhibiting $\mathrm{H}_{2} \mathrm{O}_{2}$-induced oxidative injury and cell death. In OB-6 cells, Nrf2 silencing or CRISPR/Cas9-induced Nrf2 knockout blocked 4-OIinduced osteoblast cytoprotection against $\mathrm{H}_{2} \mathrm{O}_{2}$. Conversely, forced Nrf2 activation, by CRISPR/Cas9-induced Keap1 knockout, mimicked 4-Ol-induced actions in OB-6 cells. Importantly, 4-OI was ineffective against $\mathrm{H}_{2} \mathrm{O}_{2}$ in Keap1knockout cells. Collectively, 4-Ol efficiently activates $\mathrm{Nrf2}$ signaling to inhibit $\mathrm{H}_{2} \mathrm{O}_{2}$-induced oxidative injury and death of osteoblasts.
\end{abstract}

\section{Introduction}

Sustained reactive oxygen species (ROS) production will induce profound oxidative injury to human osteoblasts ${ }^{1}$, and it is one of the primary pathology of osteoporosis and/or osteonecrosis ${ }^{2,3}$. Adding hydrogen peroxide $\left(\mathrm{H}_{2} \mathrm{O}_{2}\right)$ to cultured osteoblastic cells or primary osteoblasts is an established cellular model of osteoporosis/osteonecrosis. $\mathrm{H}_{2} \mathrm{O}_{2}$ will induce

\footnotetext{
Correspondence: Yingzi Zhang (zhanggyz@suda.edu.cn) or

Peng Cao (13601842206@163.com) or Xiangyang Xu (xxyrjhn@163.com)

'Department of Orthopedics, Ruijin Hospital, Shanghai Jiao Tong University School of Medicine, Shanghai, China

${ }^{2}$ Department of Orthopedics, Ruijin Hospital North, Shanghai Jiao Tong University School of Medicine, Shanghai, China

Full list of author information is available at the end of the article

These authors contributed equally: Yuehuan Zheng, Zhe Chen, Chang She

Edited by A. Stephanou
}

significant oxidative stress, protein damage, and DNA breaks that eventually lead to cell apoptosis and necrosis ${ }^{4-8}$. This model has been utilized to understand the mechanisms of $\mathrm{H}_{2} \mathrm{O}_{2}$-induced osteoblast cell injury and to explore possible intervention strategies ${ }^{4-8}$.

Nuclear factor E2-related factor 2 (Nrf2) is a welldefined signaling cascade that offers significant cellular protection against various oxidative stimuli ${ }^{9}$. Forced activation of Nrf2 cascade through genetic strategies or pharmacological agents can protect osteoblasts from $\mathrm{H}_{2} \mathrm{O}_{2}$ and other oxidative injury ${ }^{4,6,8,10,11}$. Wang et al. have shown that MIND4-17 was able to activate Nrf2 signaling cascade to protect OB-6 human osteoblastic cells and primary osteoblasts from $\mathrm{H}_{2} \mathrm{O}_{2}{ }^{8}$. Han et al. demonstrated that chlorogenic acid could activate PI3K-Akt-dependent

\section{(-) The Author(s) 2020}

(c) (i) Open Access This article is licensed under a Creative Commons Attribution 4.0 International License, which permits use, sharing, adaptation, distribution and reproduction cc. in any medium or format, as long as you give appropriate credit to the original author(s) and the source, provide a link to the Creative Commons license, and indicate if changes were made. The images or other third party material in this article are included in the article's Creative Commons license, unless indicated otherwise in a credit line to the material. If material is not included in the article's Creative Commons license and your intended use is not permitted by statutory regulation or exceeds the permitted use, you will need to obtain permission directly from the copyright holder. To view a copy of this license, visit http://creativecommons.org/licenses/by/4.0/. 
Nrf2 signaling and inhibit $\mathrm{H}_{2} \mathrm{O}_{2}$-induced oxidative injury in MC3T3-E1 osteoblastic cells ${ }^{10}$. MicroRNA-455 (miR455), which silenced Cullin 3, protected hFOB1 19 osteoblast cells and primary human osteoblasts from $\mathrm{H}_{2} \mathrm{O}_{2}$ by causing Nrf2 protein stabilization and Nrf2 cascade activation ${ }^{6}$. Therefore, the activation of Nrf2 cascade is a fine strategy to protect osteoblasts/osteoblastic cells from $\mathrm{H}_{2} \mathrm{O}_{2}$.

In the unstimulated resting condition, Nrf2 localizes in the cytoplasm and binds to Keap1 (Kelch-like ECHassociated protein 1$)^{12,13}$, and the latter dictates Nrf2 proteasome degradation through Cullin 3 ubiquitin complex. Once activated Nrf2 departs from Keap1, Nrf2 protein would undergo stabilization and cytosol accumulation. Stabilized Nrf2 protein translocates to the cell nuclei and binds to antioxidant-response elements (ARE), which then promotes transcription and mRNA expression of antioxidant genes and detoxifying enzymes ${ }^{12,13}$. Nrf2ARE-dependent genes include heme oxygenase 1 (HO1), $\gamma$-glutamyl cysteine ligase catalytic subunit (GCLC), NAD (P)H quinone oxidoreductase-1 (NQO1), glutathione $(G S H)$, and many others. All of them exert significant antioxidant and cytoprotective functions ${ }^{12,13}$.

Recent studies have discovered itaconate as a novel Nrf2 activator $^{14,15}$. Itaconate alkylates Keap1's key cysteine residues to activate Nrf2 signaling cascade by causing Nrf2-Keap1 disassociation and Nrf2 protein stabilization $^{14}$. A cell-permeable itaconate derivative, 4-octyl itaconate (4-OI), was synthesized and it efficiently activated Nrf2 signaling in mammalian cells ${ }^{14,16-18}$. The potential activity of 4-OI in osteoblasts has not been studied fully. Here, we reported that the activation of Nrf2 cascade by 4-OI was able to protect osteoblasts from $\mathrm{H}_{2} \mathrm{O}_{2}$-induced oxidative injury and cell death.

\section{Materials and methods}

\section{Reagents, chemicals, and antibodies}

Four-octyl itaconate (4-OI) was synthesized by Ruilu Chemicals (Shanghai, China). $\mathrm{H}_{2} \mathrm{O}_{2}$ was provided by Sigma Aldrich Chemicals (St Louis, Mo). Antibodies for HO1 (\#70081), NQO1 (\#3187), Nrf2 (\#12721), Keap1 (\#8047), Tubulin (\#2125), and Lamin B1 (\#13435), as well as cleaved-poly (ADP-ribose) polymerase (PARP, \#5625), cleaved-caspase-9 (\#20750), and immunoresponsive gene 1 (IRG1, \#77510) were obtained from Cell Signaling Tech China (Shanghai, China). The anti-GCLC antibody (ab55435) and the anti-adenine nucleotide translocase-1 (ANT1) antibody (ab102032) were purchased from Abcam China (Shanghai, China). Antibodies of PGK1 (sc130335) and cyclophilin D (CyPD, sc-137136) were provided by Santa Cruz Biotech (Santa Cruz, CA). RNA assay reagents were obtained from Thermo-Fisher Invitrogen (Suzhou, China). Cell Counting Kit-8 (CCK-8) was provided by Dojindo Laboratories (Kumamoto, Japan).

\section{Cell culture}

The differentiated OB-6 human osteoblastic cells and the primary murine osteoblasts were provided by Dr. $\mathrm{Ji}^{19,20}$. 4-OI was dissolved in DMSO in the stock solution. To test its cytoprotective activity, OB-6 osteoblastic cells or primary murine osteoblasts were pretreated with 4-OI (with final DMSO concentration at $0.2 \%$ ) for $2 \mathrm{~h}$, followed by $\mathrm{H}_{2} \mathrm{O}_{2}$ stimulation. Cells were routinely subjected to mycoplasma and microbial contamination examination every $2-3$ months. STR profiling, population doubling time, and morphology were regularly checked to confirm the genotype. The protocols were approved by the Ethics Committee of Ruijin Hospital, Shanghai Jiao Tong University School of Medicine, according to the principles of Declaration of Helsinki.

\section{Quantitative real-time PCR (qPCR)}

Following treatment, the total cellular RNA was extracted by TRIzol reagents ${ }^{21}$. The $\mathrm{qPCR}$ procedures using a SYBR Green PCR kit (Applied Biosystems, Suzhou, China) under the ABI Prism7500 Fast Real-Time PCR system were reported before ${ }^{22}$. To calculate the product melting temperature, the melting curve analysis was carried out. Glyceraldehyde-3-phosphate dehydrogenase $(G A P D H)$ was examined as the internal control and reference gene. Quantification was through the $2^{-\triangle \Delta C \mathrm{t}}$ method. The mRNA primers for Nrf2, HO1, NQO1, GCLC, and GAPDH were provided by $\mathrm{Dr} . \mathrm{Di}^{18}$. The primers of immunoresponsive gene 1 (IRG1) were purchased from OriGene (Beijing, China).

\section{Antioxidant-response element (ARE) reporter activity}

Osteoblastic cells/osteoblasts were seeded into six-well plates and transfected with an ARE-inducible firefly luciferase vector (provided by Dr. Jiang ${ }^{22}$ ). Following the applied treatments, ARE firefly luciferase activity was tested by the luminescence.

\section{Western blotting}

The detailed protocols for western blotting were described before ${ }^{22}$. An ImageJ software (from NIH) was utilized for data quantification. The same set of lysates were run in parallel ("sister") gels. To separate cell nuclei, a nuclei isolation kit (from Sigma) was applied ${ }^{23}$.

\section{Co-immunoprecipitation (Co-IP)}

A total of $700 \mu \mathrm{g}$ of protein lysates from OB-6 cells were pre-cleared with A/G beads (Sigma). The lysates were then incubated with anti-Keap1 antibody overnight ${ }^{11}$. The Keap1-bound proteins were captured by protein IgA/G beads, and examined by western blotting.

\section{Mitochondrial immunoprecipitation (Mito-IP)}

OB-6 cells were seeded into six-well tissue-culture plates. The detailed protocols of isolating mitochondrial fraction lysates and mito-IP were described before ${ }^{24}$. 


\section{Alkaline phosphatase (ALP) assay}

The primary murine osteoblasts were seeded into 12well plates $\left(6 \times 10^{4}\right.$ cells per well). Murine osteoblasts were stained with ALP using an ALP staining Kit (Beyotime Institute of Biotechnology, Wuxi, China) and cultured for 12 days. The representative ALP staining image is shown.

\section{Cell viability}

OB-6 osteoblastic cells or primary murine osteoblasts were seeded into 96-well plates (at $2 \times 10^{3}$ cells per well). Following treatment, a CCK-8 assay kit was utilized to quantify cell viability, with CCK-8's optical density (OD) examined at $490 \mathrm{~nm}$.

\section{Cell apoptosis-related assays}

Cell apoptosis-related assays, including the caspase-3 activity assay, Annexin V-propidium iodide (PI) FACS, and nuclear terminal deoxynucleotidyl transferase dUTP nick-end labeling (TUNEL) staining were described in other studies ${ }^{23,25,26}$. For TUNEL staining, TUNEL/DAPI ratio was calculated from recording at least 500 cells from five random microscope views $(1 \times 100)$.

\section{Mitochondrial depolarization}

With cells undergoing mitochondrial depolarization, the JC-1 fluorescence dye (Sigma) accumulates in the mitochondria to form green monomers ${ }^{27}$. The detailed protocols for JC-1 assay of mitochondrial depolarization have been described before ${ }^{11}$. The JC-1 fluorescence images with merging green and red fluorescence channels are presented.

\section{ROS detection}

OB-6 osteoblastic cells or primary murine osteoblasts were seeded into 12-well tissue-culture plates. After treatment, cells were stained with CellROX $(5 \mu \mathrm{M}$, Invitrogen-Thermo Fisher). CellROX intensity was tested by a fluorescent spectrophotometer. CellROX images were represented.

\section{Glutathione contents}

OB-6 cells or primary murine osteoblasts were seeded into six-well plates. The ratio of reduced glutathione $(\mathrm{GSH})$ to the oxidized disulfide form glutathione (GSSG) ${ }^{28}$ was tested using a previously described protocol ${ }^{28}$.

\section{Single-strand DNA (ssDNA) ELISA}

OB-6 cells or primary murine osteoblasts were seeded into six-well plates. Following treatment, the cellular ssDNA contents were examined by an ApoStrandTM ELISA kit (BIOMOL International, Plymouth Meeting, PA). The ssDNA ELISA OD was recorded at $450 \mathrm{~nm}$.

\section{Lactate dehydrogenase (LDH) assay}

OB-6 cells or primary murine osteoblasts were seeded into 12 -well tissue-culture plates $\left(6 \times 10^{4}\right.$ cells per well). Following treatment, a two-step simple LDH assay kit (Takara, Tokyo, Japan) was utilized to quantify LDH contents in the medium, which were then normalized to total LDH contents.

\section{Nrf2 short-hairpin RNA (shRNA)}

OB-6 cells were seeded into six-well plates and transfected with the Nrf2 shRNA lentiviral particles (sc37030V, Santa Cruz Biotech, Santa Cruz, CA). To select stable cells, puromycin $(2.5 \mu \mathrm{g} / \mathrm{mL})$ was added in the complete medium (for 4-5 passages). Nrf2 silencing in the stable cells, namely, sh-Nrf2 cells, was confirmed by western blotting and qPCR analyses.

\section{CRISPR-Cas9-mediated gene knockout (KO)}

OB-6 cells were transduced with a CRISPR/Cas9-Nrf2KO-GFP-puro construct or a CRISPR/Cas9-Keap1-KOGFP-puro construct provided by Dr. Liu ${ }^{18}$. The GFPpositive transfected cells were sorted by FACS. The selected cells were distributed into 96-well plates and cultured in puromycin $(2.5 \mu \mathrm{g} / \mathrm{mL})$-containing medium. In stable cells, Nrf2 $\mathrm{KO}$ or Keap1 $\mathrm{KO}$ was verified by western blotting and qPCR analyses.

\section{Forced expression of IRG1}

The full-length human IRG1 cDNA (provided by Genechem) was sub-cloned into a GV369 lentiviral construct (Genechem, Shanghai, China). The construct was transfected to HEK-293 cells together with the lentiviruspacking helper plasmids (Genechem) to generate the IRG1-expressing lentivirus. The lentivirus was added to OB-6 cells (cultured in polybrene-containing complete medium). Following selection by puromycin $(2.5 \mu \mathrm{g} / \mathrm{ml}$, for 5-6 passages), stable cells were established: OE-IRG1 cells. IRG1 overexpression was verified by western blotting and qPCR analyses.

\section{Itaconate contents}

OB-6 cells were seeded into six-well tissue-culture plates. The cellular itaconate contents were tested by a commercial kit from Biyuntian (Wuxi, China) using the attached protocol. The levels were normalized to control.

\section{Statistical analysis}

The investigators were blinded to group allocation. Quantitative data with normal distribution were always presented as mean \pm standard deviation (SD). Statistical analyses were performed by two-way ANOVA using a Scheffe's $F$ test (SPSS 23.0, SPSS Co.. Chicago, IL). To test significance between two treatment groups, a two-tailed 
unpaired $T$ test (Excel 2007) was utilized. $P<0.05$ was considered as statistically significant.

\section{Results}

Nrf2 cascade activation by 4-OI in osteoblasts

We first tested whether 4-OI can provoke Nrf2 signaling cascade in human osteoblasts. The coimmunoprecipitation (Co-IP) assay results in Fig. 1a demonstrated that Keap1 immunoprecipitated with Nrf2 in the vehicle $(0.2 \%$ of DMSO)-treated control OB-6 human osteoblastic cells ${ }^{8,29}$. The Keap1-Nrf2 association was disrupted with stimulation of 4-OI (Fig. 1a). 4-OI was utilized at $10-25 \mu \mathrm{M}$, referring other studies ${ }^{14,16,18}$. Following disassociation with Keap1, the Nrf2 protein was stabilized and accumulated in 4-OI-treated OB-6 cells (Fig. 1b). Keap1 protein expression was unchanged (Fig. 1b). Nrf2 protein elevation by 4-OI was unlikely due to de novo protein synthesis since Nrf2 mRNA levels were not significantly increased (Fig. 1c). As Nrf2 protein levels were dramatically increased in the nuclei fraction lysates of 4-OI-treated OB-6 cells, stabilized Nrf2 protein translocated to cell nuclei (Fig. 1d). Importantly, the relative reporter activity of ARE was robustly elevated following 4OI treatment in OB-6 cells (Fig. 1e), resulting in the increased transcription and mRNA expression of Nrf2ARE-dependent genes: HO1, NQO1, and GCLC (Fig. 1f). Protein expressions of HO1, NQO1, and GCLC were significantly upregulated as well in 4-OI-treated OB-6 cells (Fig. 1g). These results clearly showed that 4-OI is able to activate Nrf2 signaling cascade in OB-6 cells. Notably, 4-OI displayed a concentration-dependent activity as it was more efficient in activating Nrf2 cascade at $25 \mu \mathrm{M}$ than at $10 \mu \mathrm{M}$ (Fig. 1a-g).

In the primary murine osteoblasts (the morphology with ALP staining shown in Fig. 1h), treatment with 4-OI $(25 \mu \mathrm{M})$ activated Nrf2 cascade, leading to Nrf2 protein stabilization (Fig. 1h), and increased mRNA and protein expression of Nrf2-ARE-dependent genes, HO1, NQO1, and $G C L C$ (Fig. 1h, i).

\section{$\mathrm{H}_{2} \mathrm{O}_{2}$-induced oxidative injury is inhibited by $4-\mathrm{Ol}$ in osteoblasts}

$\mathrm{H}_{2} \mathrm{O}_{2}$ stimulation in cultured osteoblasts/osteoblastic cells would induce oxidative stress and cause profound protein damage, lipid peroxidation, and DNA breaks $^{4,8,30,31}$. In human osteoblastic OB-6 cells, $\mathrm{H}_{2} \mathrm{O}_{2}$ $(400 \mu \mathrm{M})$ significantly increased ROS production, evidenced by the increased CellROX intensity ${ }^{32,33}$ (Fig. 2a). Further confirming the oxidative injury, the GSH/GSSG ratio was decreased following $\mathrm{H}_{2} \mathrm{O}_{2}$ stimulation in OB-6 cells (Fig. 2b). Additionally, levels of single-strand DNA (ssDNA) were increased in $\mathrm{H}_{2} \mathrm{O}_{2}$-treated OB-6 cells (Fig. 2c). Significantly, pretreatment with 4-OI (at $10 / 25 \mu \mathrm{M}$ ) largely inhibited $\mathrm{H}_{2} \mathrm{O}_{2}$-induced oxidative injury in OB-6 cells, and suppressed CellROX intensity increase (Fig. 2a), GSH/GSSG ratio reduction (Fig. 2b), and ssDNA accumulation (Fig. 2c).

Similar results were detected in primary murine osteoblasts as well, since 4-OI $(25 \mu \mathrm{M})$ pretreatment significantly attenuated $\mathrm{H}_{2} \mathrm{O}_{2}$-induced $\mathrm{ROS}$ production (CellROX intensity increase, Fig. 2d), the GSH/GSSG ratio reduction (Fig. 2e), and ssDNA accumulation (Fig. 2f). Importantly, 4-OI single treatment failed to alter basal oxidative levels in OB-6 cells (Fig. 2a-c) and primary murine osteoblasts (Fig. 2d-f).

$\mathrm{H}_{2} \mathrm{O}_{2}$-induced apoptosis is inhibited by $4-\mathrm{OI}$ in osteoblasts

Next, we studied whether 4-OI can inhibit $\mathrm{H}_{2} \mathrm{O}_{2}$ induced cytotoxicity in osteoblasts. CCK- 8 viability assay results demonstrated that in OB-6 osteoblastic cells, $\mathrm{H}_{2} \mathrm{O}_{2}$ $(400 \mu \mathrm{M})$ treatment potently decreased CCK-8 OD (Fig. 3a). This was largely attenuated by pretreatment of 4-OI $(10 / 25 \mu \mathrm{M})$ (Fig. 3a). $\mathrm{H}_{2} \mathrm{O}_{2}$-induced caspase-3 activation (Fig. 3b) as well as cleavages of caspase- 9 and poly (ADPribose) polymerase (PARP) (Fig. 3c) were both inhibited with 4-OI pretreatment. $\mathrm{H}_{2} \mathrm{O}_{2}$ provoked apoptosis activation, increased TUNEL-positive nuclei ratio (Fig. 3d), and the number of Annexin V-gated cells (Fig. 3e) in OB6 osteoblastic cells. Again, 4-OI pretreatment inhibited $\mathrm{H}_{2} \mathrm{O}_{2}$-induced apoptosis activation in OB-6 cells (Fig. 3d, e). Treatment with 4-OI alone failed to induce caspase and apoptosis activation in OB-6 cells (Fig. 3a-e).

In primary murine osteoblasts, $4-\mathrm{OI}(25 \mu \mathrm{M})$ pretreatment attenuated $\mathrm{H}_{2} \mathrm{O}_{2}$-induced viability reduction (Fig. $3 \mathrm{f}$ ). $\mathrm{H}_{2} \mathrm{O}_{2}$ provoked caspase- 3 activation (Fig. $3 \mathrm{~g}$ ) and apoptosis induction (Fig. 3h, i). Apoptosis activation was evidenced by increased nuclear TUNEL staining (Fig. 3h) and an elevated number of Annexin V-gated cells (Fig. 3i). Significantly, 4OI pretreatment largely attenuated $\mathrm{H}_{2} \mathrm{O}_{2}$-induced apoptosis activation in primary murine osteoblasts (Fig. $3 \mathrm{~g}-\mathrm{i}$ ). Together, these results showed that 4-OI inhibited $\mathrm{H}_{2} \mathrm{O}_{2}$ induced cytotoxicity and apoptosis in osteoblasts.

\section{$\mathrm{H}_{2} \mathrm{O}_{2}$-induced programmed necrosis is inhibited by $4-\mathrm{Ol}$ in osteoblasts}

Besides apoptosis, $\mathrm{H}_{2} \mathrm{O}_{2}$ and oxidative stress could also induce programmed necrosis in osteoblasts ${ }^{6,30,34,35}$. Upon programmed necrosis, p53 would translocate to the mitochondria to form a complex with mPTP components cyclophilin $\mathrm{D}(\mathrm{CyPD})$ and adenine nucleotide translocase 1 $(\mathrm{ANT} 1)^{36-38}$. This then leads to the opening of mitochondrial permeability transition pore (mPTP) and mitochondrial depolarization, and finally cell necrosis ${ }^{36-38}$. By applying the mito-immunoprecipitation (mito-IP) assay, we showed that CyPD immunoprecipitated with ANT1 and p53 in the mitochondria of $\mathrm{H}_{2} \mathrm{O}_{2}$-treated OB-6 osteoblastic cells (Fig. 4a). Tested by the mitochondrial accumulation of JC-1 green monomers, we also detected mitochondrial 


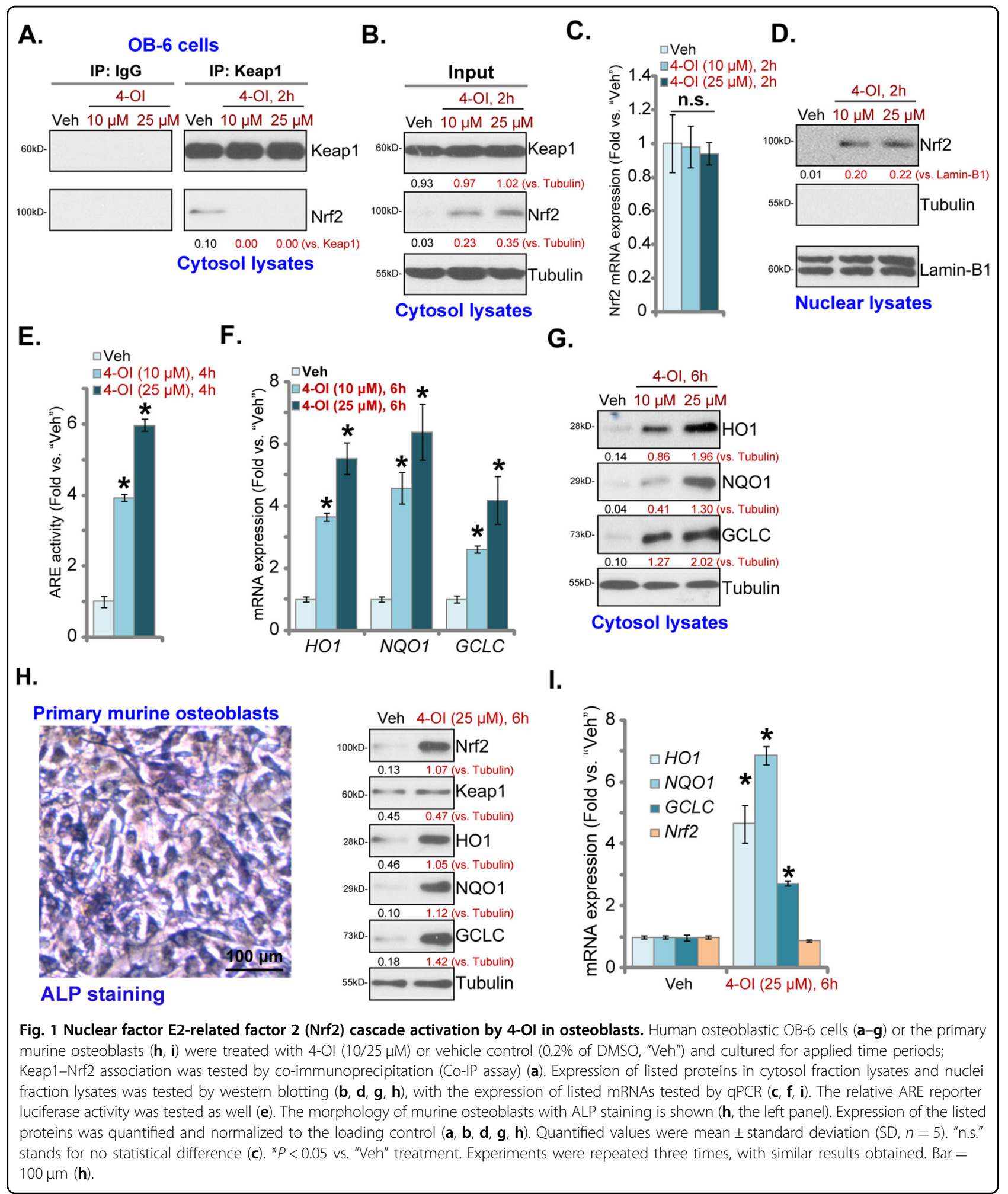

depolarization in these cells (Fig. 4b). Importantly, 4-OI (10/ $25 \mu \mathrm{M})$ pretreatment largely inhibited $\mathrm{H}_{2} \mathrm{O}_{2}$-induced CyPD-ANT1-p53 association (Fig. 4a) and mitochondrial depolarization (Fig. 4b) in OB-6 cells. Expressions of CyPD,
ANT1 and p53 proteins in mitochondrial lysates were however unchanged (Fig. 4a, "inputs").

Further studies confirmed that that $\mathrm{H}_{2} \mathrm{O}_{2}(400 \mu \mathrm{M})$ treatment induced OB-6 cell necrosis by causing 
A.

OB-6 cells
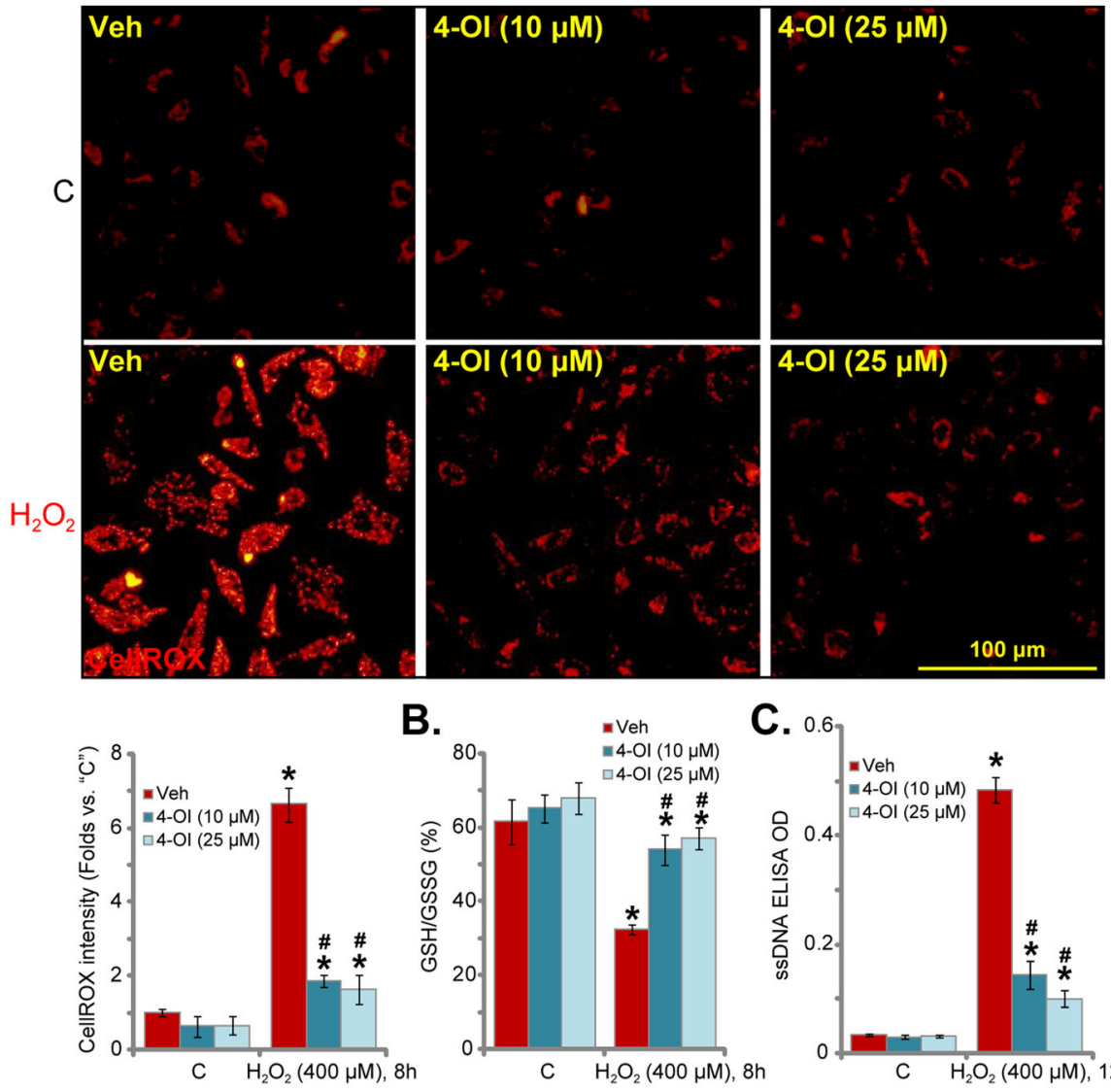

B.
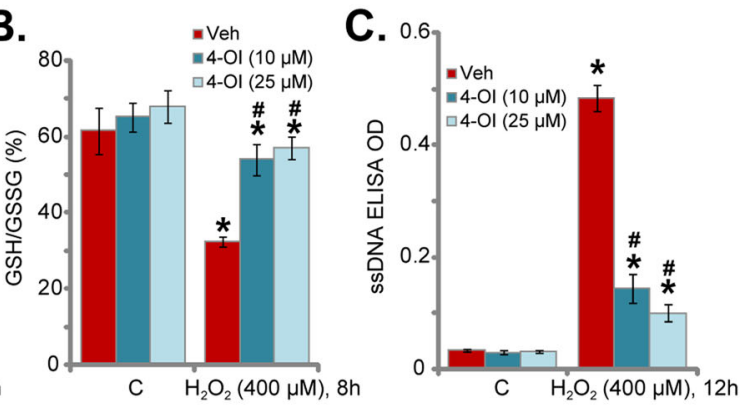

D.

E.

\section{F.}

Primary murine osteoblasts
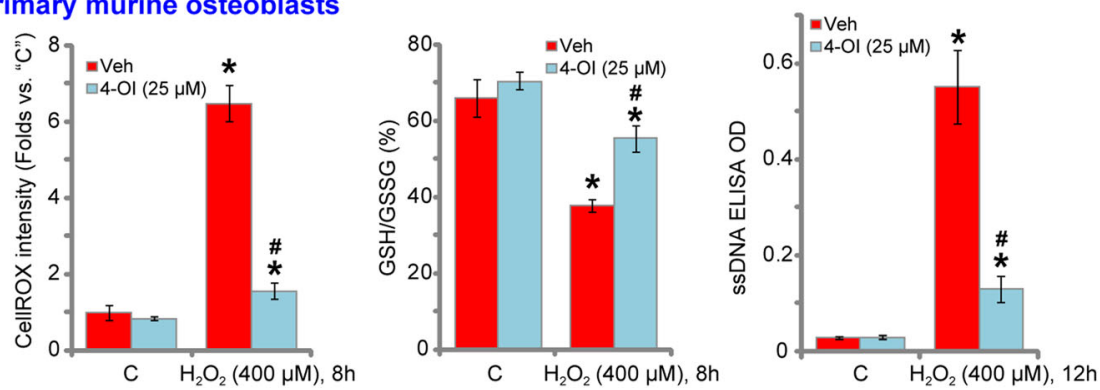

Fig. $2 \mathrm{H}_{2} \mathrm{O}_{2}$-induced oxidative injury was inhibited by $4-\mathrm{Ol}$ in osteoblasts. Human osteoblastic OB-6 cells (a-c) or primary murine osteoblasts (d-f) were pretreated for $2 \mathrm{~h}$ with 4-OI $(10 / 25 \mu \mathrm{M})$ or vehicle control ("Veh"), followed by $\mathrm{H}_{2} \mathrm{O}_{2}(400 \mu \mathrm{M})$ stimulation. Cells were further cultured for applied time periods, reactive oxygen species (ROS) production (tested by CellROX intensity, a, d), the GSH/GSSG ratio (b, e), and single-strand DNA (ssDNA) contents (ELISA OD, $\mathbf{c}, \mathbf{f}$ ) were tested. Quantified values were mean \pm standard deviation $(S D, n=5) .{ }^{~} C^{\prime \prime}$ stands for the untreated control cells. ${ }^{*} P<0.05$ vs. "C" cells. ${ }^{\#} P<0.05$ vs. cells with $\mathrm{H}_{2} \mathrm{O}_{2}$ stimulation but "Veh" pretreatment. Experiments were repeated three times, with similar results obtained. Bar $=100 \mu \mathrm{m}(\mathbf{a}, \mathbf{d})$.

significant LDH release to the culture medium (Fig. 4c), which was largely attenuated by 4-OI pretreatment (Fig. 4c). In the primary murine osteoblasts, mitochondrial depolarization (indicated by JC-1 green monomer fluorescence increase, Fig. 4d) and cell necrosis (medium LDH release, Fig. 4e) were detected following $\mathrm{H}_{2} \mathrm{O}_{2}$ stimulation. Again, such actions were largely inhibited by 4-OI $(25 \mu \mathrm{M})$ pretreatment (Fig. $4 \mathrm{~d}$, e). These results indicated that 4-OI is able to inhibit $\mathrm{H}_{2} \mathrm{O}_{2}$-induced programmed necrosis in osteoblasts. 


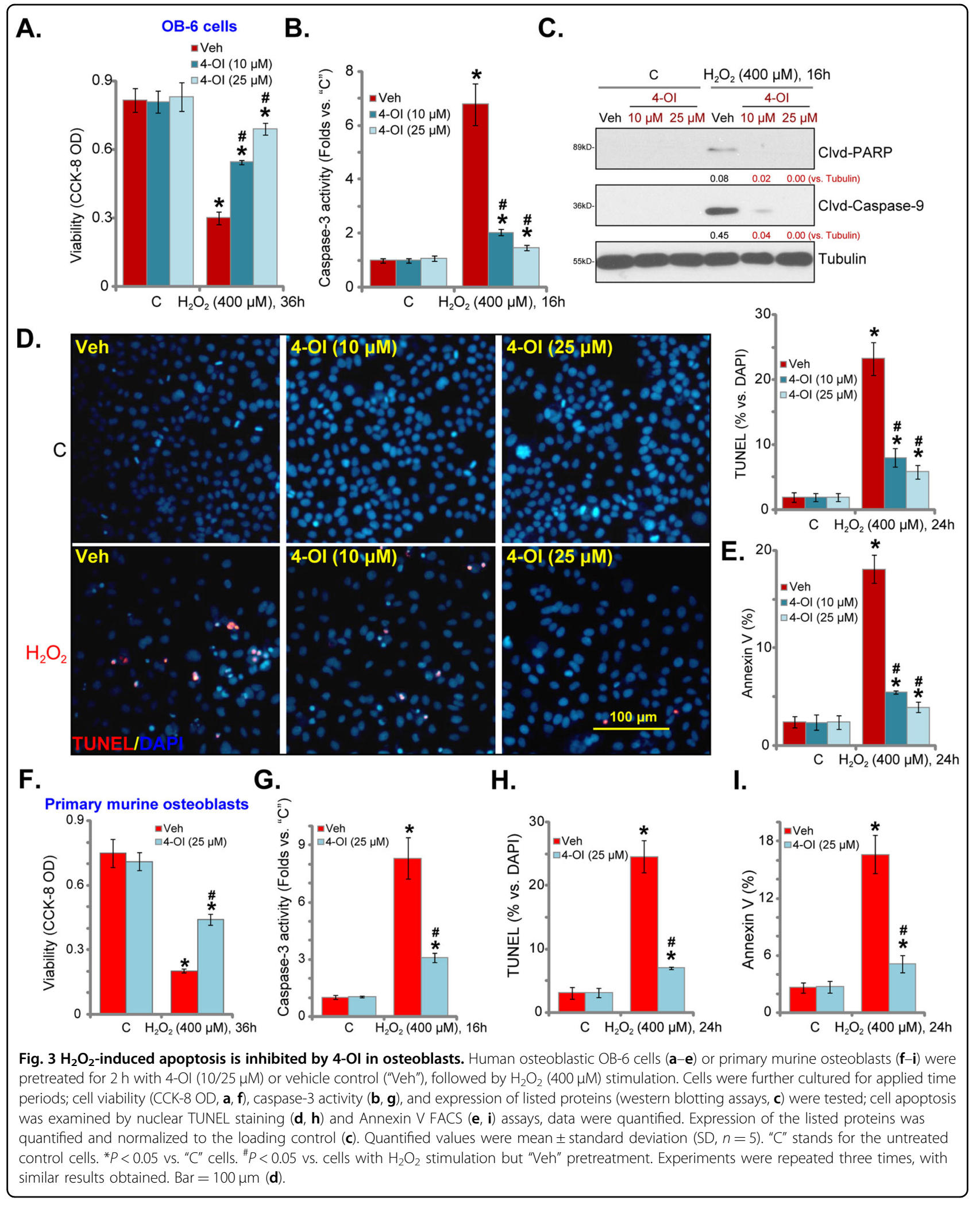


A.

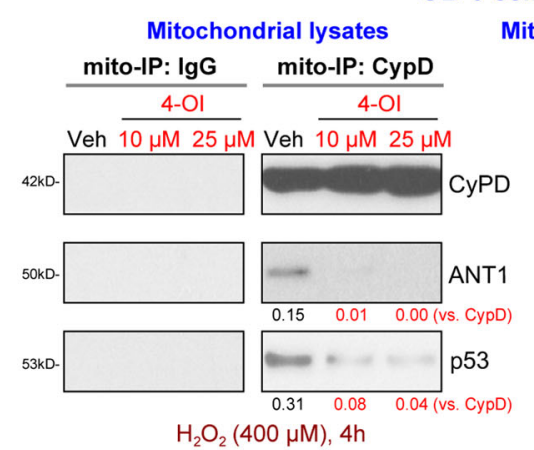

B.

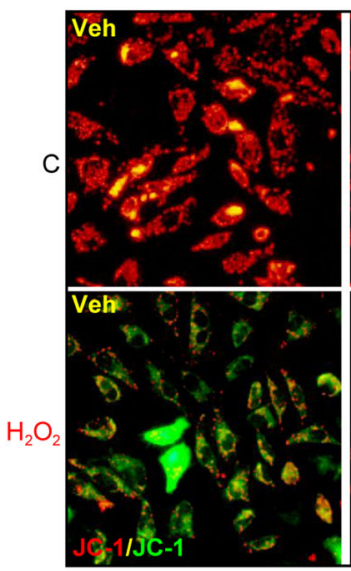

D.

Primary murine osteoblasts

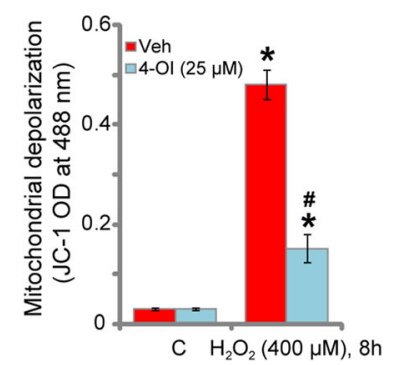

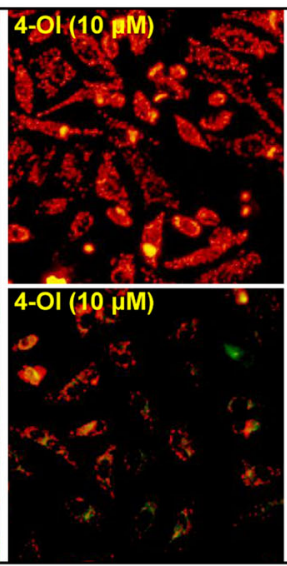

E.

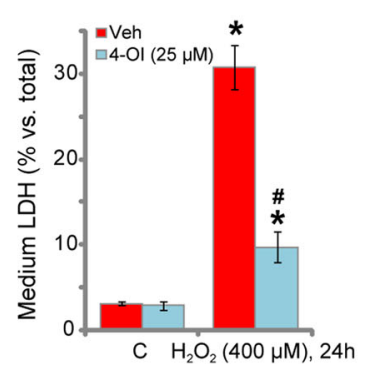

C.
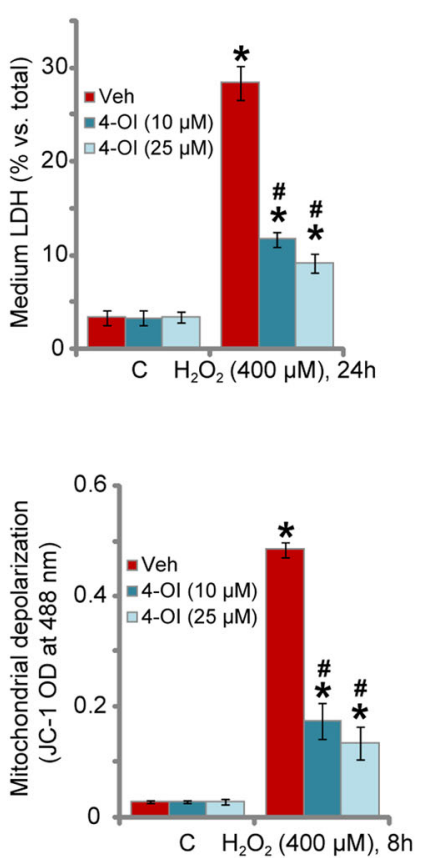

Fig. $4 \mathrm{H}_{2} \mathrm{O}_{2}$-induced programmed necrosis is inhibited by $4-\mathrm{OI}$ in osteoblasts. Human osteoblastic OB-6 cells (a-c) or the primary murine osteoblasts (d, e) were pretreated for $2 \mathrm{~h}$ with 4-Ol (10/25 $\mu \mathrm{M})$ or vehicle control ("Veh"), followed by $\mathrm{H}_{2} \mathrm{O}_{2}(400 \mu \mathrm{M})$ stimulation. Cells were further cultured for applied time periods; mitochondrial CyPD-ANT1-p53 association and their expression are shown (a); mitochondrial depolarization was tested by JC-1 green monomer fluorescence $(\mathbf{b}, \mathbf{d})$, and cell necrosis examined by quantifying medium LDH release (c, e). Expression of the listed proteins was quantified and normalized to the loading control (a). Quantified values were mean \pm standard deviation $(\mathrm{SD}, n=5)$. " $\mathrm{C}$ " stands for the untreated control cells. ${ }^{*} P<0.05$ vs. " $\mathrm{C}$ " cells. ${ }^{\#} P<0.05$ vs. cells with $\mathrm{H}_{2} \mathrm{O}_{2}$ stimulation but "Veh" pretreatment. Experiments were repeated three times, with similar results obtained. $\mathrm{Bar}=100 \mu \mathrm{m}(\mathbf{b})$.

Forced overexpression of IRG1 activates Nrf2 signaling and protects osteoblasts from $\mathrm{H}_{2} \mathrm{O}_{2}$

We next hypothesized that forced overexpression of IRG1, a rate-limiting enzyme in itaconate synthesis ${ }^{14}$, might increase endogenous itaconate production to activate Nrf2 cascade. A lentiviral IRG1 expression construct was transduced to OB-6 osteoblastic cells. Stable cells were established with FACS-mediated GFP sorting and puromycin selection: namely, OE-IRG1 cells. As shown, IRG1 mRNA and protein levels were significantly increased in OE-IRG1 cells (vs. vector control cells, Fig. 5a). As a result, the cellular itaconate contents increased over tenfolds of vector control cells (Fig. 5b). Overexpression of IRG1 induced Nrf2 protein stabilization 


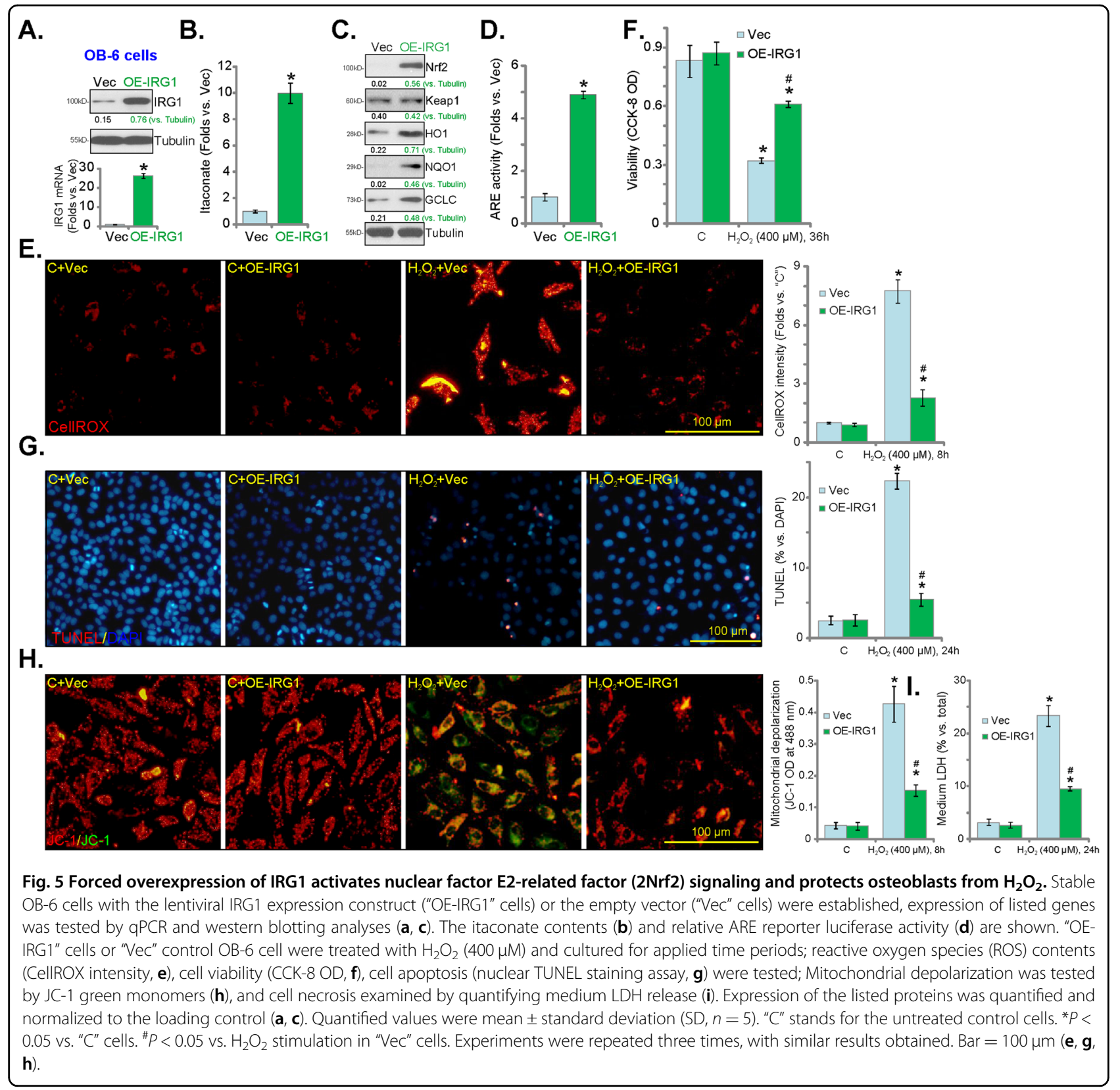

(Fig. 5c), and also increased the expression of HO1, NQO1, and GCLC proteins (Fig. 5c) in OB-6 cells. The relative ARE reporter luciferase activity was boosted, as well in the OE-IRG1 cells (Fig. 5d). Therefore, IRG1 overexpression increased itaconate production and activated Nrf2 signaling in OB-6 cells.

By analyzing CellROX fluorescence intensity, we demonstrated that $\mathrm{H}_{2} \mathrm{O}_{2}$-induced $\mathrm{ROS}$ production was largely inhibited in OE-IRG1 cells (Fig. 5e). Furthermore, $\mathrm{H}_{2} \mathrm{O}_{2}$-induced viability reduction was attenuated in IRG1overexpressed OB-6 cells (Fig. 5f). TUNEL assay results showed that with IRG1 overexpression, $\mathrm{H}_{2} \mathrm{O}_{2}$-induced apoptosis activation was potently inhibited (Fig. 5g).
Additional studies demonstrated that $\mathrm{H}_{2} \mathrm{O}_{2}$-induced mitochondrial depolarization (JC-1 green monomers accumulation, Fig. 5h) and cell necrosis (medium LDH release, Fig. 5i) were inhibited in OB-6 cells bearing IRG1 expression construct. These results showed that exogenous IRG1 overexpression attenuated $\mathrm{H}_{2} \mathrm{O}_{2}$-induced apoptosis and programmed necrosis in OB-6 cells.

\section{Nrf2 cascade activation mediates 4-OI-induced osteoblast cytoprotection against $\mathrm{H}_{2} \mathrm{O}_{2}$}

If 4-OI-induced osteoblast cytoprotection against $\mathrm{H}_{2} \mathrm{O}_{2}$ required Nrf2 cascade activation, it should be ineffective in Nrf2-depleted osteoblasts. To test this hypothesis, 
Nrf2 shRNA lentivirus was transfected into OB-6 cells, and stable cells (sh-Nrf2 cells) were established with puromycin selection. Alternatively, OB-6 cells were transduced with the CRISPR/Cas9-Nrf2-GFP-KO construct ${ }^{18}$. With FACS-mediated GFP sorting and puromycin selection, stable cells were established: namely, ko-Nrf2 cells. Control cells were transduced with scramble control shRNA and CRISPR/Cas9 empty vector: sh-C +Cas9-C cells. In sh-Nrf2 cells and ko-Nrf2 cells, 4-OIinduced Nrf2 protein stabilization (observed in sh-C +Cas9-C cells) was completely reversed (Fig. 6a). Additionally, mRNA and protein expressions of Nrf2-AREdependent genes (HO1, NQO1, and GCLC) by 4-OI were blocked with Nrf2 shRNA or $\mathrm{KO}$ (Fig. 6a, b). $\mathrm{H}_{2} \mathrm{O}_{2}$ induced cell death (CCK-8 OD reduction, Fig. 6c) and apoptosis (nuclear TUNEL staining, Fig. 6d) were intensified in sh-Nrf2 cells and ko-Nrf2 cells (vs. sh-C+Cas9-C cells). Importantly, 4-OI was unable to inhibit $\mathrm{H}_{2} \mathrm{O}_{2}$ induced cytotoxicity in Nrf2 silencing or KO OB-6 cells (Fig. 6c, d). Therefore, Nrf2 depletion reversed 4-OIinduced osteoblast cytoprotection against $\mathrm{H}_{2} \mathrm{O}_{2}$.

We further hypothesized that forced activation of Nrf2 should mimic 4-OI-induced osteoblast cytoprotection. A CRISPR/Cas9-Keap1-GFP-KO construct ${ }^{18}$ was transduced to OB-6 osteoblastic cells. Stable cells were again established with FACS sorting and puromycin selection: namely, ko-Keap1 cells. Keap1 KO induced significant Nrf2 cascade activation, led to Nrf2 protein accumulation (Fig. 6e), as well as increased expression of Nrf2-ARE-dependent genes ( $\mathrm{HO1}, \mathrm{NQO1}$, and GCLC) (Fig. 6e, f). The ko-Keap1 cells were resistant to $\mathrm{H}_{2} \mathrm{O}_{2}$ induced cell death (Fig. 6g) and apoptosis (Fig. 6h). Importantly, in ko-Keap1 cells, 4-OI treatment failed to increase Nrf2 cascade activation (Fig. 6e, f). It did not offer further osteoblast cytoprotection against $\mathrm{H}_{2} \mathrm{O}_{2}$ (Fig. $6 \mathrm{~g}$, h). These results further supported that $\mathrm{Nrf2}$ cascade activation mediated 4-OI-induced osteoblast cytoprotection against $\mathrm{H}_{2} \mathrm{O}_{2}$.

\section{Discussion}

Activation of Nrf2 cascade can protect osteoblastic cells and primary osteoblasts from $\mathrm{H}_{2} \mathrm{O}_{2}$ and other oxidative stimuli ${ }^{6,8,10}$. Following activation, Nrf2 protein separates from Keap1, translocates to cell nuclei, and binds with ARE to promote transcription and mRNA expression of antioxidant and detoxifying genes, including HO1, GSH, NQO1, and GCLC $C^{39-41}$. These genes then exert significant ROS scavenging and antioxidant activities $^{9,42,43}$.

Alkylating Keap1's cysteine residues by itaconate can cause Nrf2-Keap1 departure and Nrf2 cascade activation $^{14,18}$. Recent studies have developed 4-OI, the cellpermeable itaconate derivative, as a potent and efficient Nrf2 activator. Liu et al. showed that 4-OI activated Nrf2 cascade to protect neuronal cells from $\mathrm{H}_{2} \mathrm{O}_{2}{ }^{18}$. Activation of Keap1-Nrf2 signaling by 4-OI protected human umbilical vein endothelial cells from high glucose-induced oxidative injure and cell apoptosis as well ${ }^{16}$. In peripheral blood mononuclear cells of systemic lupus erythematosus patients, 4-OI activated Nrf2 signaling to inhibit proinflammatory cytokines production ${ }^{17}$.

Here in OB-6 cells and primary murine osteoblasts, 4OI activated Nrf2 signaling cascade that led to Keap1-Nrf2 disassociation, Nrf2 protein stabilization, cytosol accumulation, and nuclear translocation. It also increased ARE reporter activity as well as mRNA and protein expression of ARE-dependent genes (HO1, $N Q O 1$, and GCLC). Functional studies showed that 4-OI pretreatment largely inhibited $\mathrm{H}_{2} \mathrm{O}_{2}$-induced ROS production and oxidative injury in both OB-6 osteoblastic cells and primary murine osteoblasts. Furthermore, $\mathrm{H}_{2} \mathrm{O}_{2}$ cell death and apoptosis in osteoblasts were largely attenuated with 4-OI pretreatment.

Existing studies have shown that $\mathrm{mPTP}$ is essential in mediating $\mathrm{H}_{2} \mathrm{O}_{2}$-induced cell death ${ }^{44}$. mPTP is formed by two structural components (ANT1 and VDAC, in the inner mitochondrial membrane) as well as the regulatory component $\mathrm{CyPD}$ (at the outer mitochondrial membrane) ${ }^{36-38} \cdot \mathrm{H}_{2} \mathrm{O}_{2}$ stimulation would lead to $\mathrm{p} 53$ translocation to the mitochondria to form a complex with CyPD and ANT1, and cause MPTP opening, mitochondrial depolarization, and eventually cell necrosis ${ }^{37,38}$. The mitochondrial programmed necrosis pathway is also important for $\mathrm{H}_{2} \mathrm{O}_{2}$-induced osteoblast cell death ${ }^{34}$. Here, we showed that 4-OI inhibited $\mathrm{H}_{2} \mathrm{O}_{2}$ induced programmed necrosis by suppressing mitochondrial depolarization, mitochondrial CyPD-ANT1p53 association, and medium LDH release in OB-6 cells and primary murine osteoblasts. This should explain the superior osteoblast cytoprotective action by the Nrf2 activator.

Activation of Nrf2 cascade using genetic strategies or pharmacological agents can efficiently protect osteoblasts from $\mathrm{H}_{2} \mathrm{O}_{2}$-induced oxidative injury and cytotoxicity ${ }^{6,8,10}$. The results of this study supported that the activation of $\mathrm{Nrf2}$ is required for 4-OI-induced osteoblast cytoprotection against $\mathrm{H}_{2} \mathrm{O}_{2}$. In OB-6 cells, Nrf2 shRNA or CRISPR/ Cas9-induced Nrf2 knockout blocked 4-OI-induced osteoblast cytoprotection against $\mathrm{H}_{2} \mathrm{O}_{2}$. Conversely, ectopic overexpression of IRG1 increased endogenous itaconate production and activated Nrf2 signaling cascade, while inhibiting $\mathrm{H}_{2} \mathrm{O}_{2}$-induced oxidative injury, cell death, and apoptosis. Similarly, forced Nrf2 cascade activation by CRISPR/Cas9-induced Keap1 KO mimicked 4OI-induced actions and inhibited $\mathrm{H}_{2} \mathrm{O}_{2}$-cell death and apoptosis in OB-6 cells. Importantly, 4-OI failed to offer further osteoblast cytoprotection against $\mathrm{H}_{2} \mathrm{O}_{2}$ when $\mathrm{Nrf} 2$ was pre-activated in Keap1 $\mathrm{KO}$ cells. These results clearly 


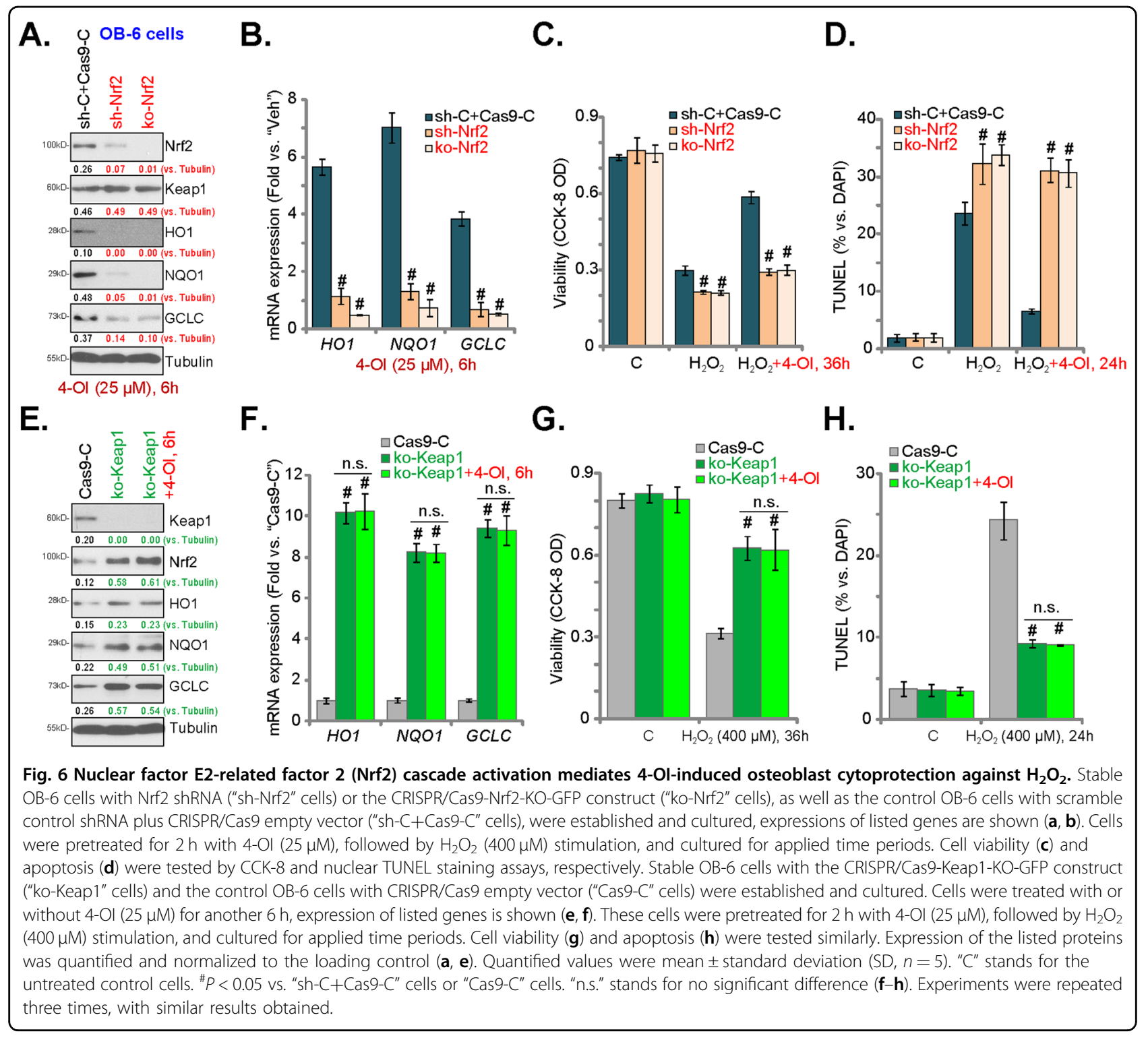

demonstrated that $\mathrm{Nrf} 2$ cascade activation by 4-OI inhibited $\mathrm{H}_{2} \mathrm{O}_{2}$-induced oxidative injury and cell death in osteoblasts.

\section{Conclusion}

Taken together, this study demonstrated that 4-OI activated Nrf2 signaling and protected osteoblasts/osteoblastic cells from $\mathrm{H}_{2} \mathrm{O}_{2}$-induced cytotoxicity. Activation of Nrf2 cascade by 4-OI could be a novel strategy to protect osteoblasts from oxidative injury.

\section{Acknowledgements}

This work is supported by the Fundings of North Hospital of Ruijin Hospital, Shanghai Jiaotong University School of Medicine (2019ZY05), Science and Technology Innovation Act of Shanghai in 2018 (18140901500), National
Science Foundation of China (81902207), and by Jiangsu Young Medical Talent Project (QNRC2016879).

\section{Author details}

${ }^{1}$ Department of Orthopedics, Ruijin Hospital, Shanghai Jiao Tong University School of Medicine, Shanghai, China. ${ }^{2}$ Department of Orthopedics, Ruijin Hospital North, Shanghai Jiao Tong University School of Medicine, Shanghai, China. ${ }^{3}$ Department of Orthopedics, The Second Affiliated Hospital of Soochow University, Suzhou, China

\section{Conflict of interest}

The authors declare that they have no conflict of interest.

\section{Publisher's note}

Springer Nature remains neutral with regard to jurisdictional claims in published maps and institutional affiliations. 
Received: 10 July 2020 Revised: 2 September 2020 Accepted: 4 September 2020

Published online: 17 September 2020

\section{References}

1. Manolagas, S. C. From estrogen-centric to aging and oxidative stress: a revised perspective of the pathogenesis of osteoporosis. Endocr. Rev. 31, 266-300 (2010).

2. Schroder, K. NADPH oxidases in bone homeostasis and osteoporosis. Free Radic. Biol. Med. 132, 67-72 (2019).

3. Frenkel, B., White, W. \& Tuckermann, J. Glucocorticoid-induced osteoporosis. Adv. Exp. Med Biol. 872, 179-215 (2015).

4. Liang, J. et al. Circular RNA HIPK3 downregulation mediates hydrogen peroxide-induced cytotoxicity in human osteoblasts. Aging 12, 1159-1170 (2020).

5. Ruan, J. W., Yao, C., Bai, J. Y. \& Zhou, X. Z. microRNA-29a inhibition induces Gab1 upregulation to protect OB-6 human osteoblasts from hydrogen peroxide. Biochem. Biophys. Res. Commun. 503, 607-614 (2018).

6. $\mathrm{Xu}, \mathrm{D}$. et al. microRNA-455 targets cullin 3 to activate Nrf2 signaling and protect human osteoblasts from hydrogen peroxide. Oncotarget $\mathbf{8}$, 59225-59234 (2017)

7. Liu, W. et al. Targeted activation of AMPK by GSK621 ameliorates $\mathrm{H}_{2} \mathrm{O}_{2}$ induced damages in osteoblasts. Oncotarget 8, 10543-10552 (2017).

8. Guo, S. et al. Activation of Nif2 by MIND4-17 protects osteoblasts from hydrogen peroxide-induced oxidative stress. Oncotarget 8, 105662-105672 (2017).

9. Suzuki, T. \& Yamamoto, M. Molecular basis of the Keap1-Nrf2 system. Free Radic. Biol. Med. 88, 93-100 (2015).

10. Han, D. et al. Cytoprotective effect of chlorogenic acid against hydrogen peroxide-induced oxidative stress in MC3T3-E1 cells through PI3K/Akt-mediated Nrf2/HO-1 signaling pathway. Oncotarget 8, 14680-14692 (2017).

11. Liang, J. et al. PGK1 depletion activates Nrf2 signaling to protect human osteoblasts from dexamethasone. Cell Death Dis. 10, 888 (2019).

12. Zhang, H., Davies, K. J. A. \& Forman, H. J. Oxidative stress response and Nrf2 signaling in aging. Free Radic. Biol. Med. 88, 314-336 (2015).

13. Keum, Y. S. \& Choi, B. Y. Molecular and chemical regulation of the Keap1-Nrf2 signaling pathway. Molecules 19, 10074-10089 (2014).

14. Mills, E. L. et al. Itaconate is an anti-inflammatory metabolite that activates Nrf2 via alkylation of KEAP1. Nature 556, 113-117 (2018).

15. Bambouskova, M. et al. Electrophilic properties of itaconate and derivatives regulate the IkappaBzeta-ATF3 inflammatory axis. Nature 556, 501-504 (2018).

16. Tang, C., Tan, S., Zhang, Y., Dong, L. \& Xu, Y. Activation of Keap1-Nrf2 signaling by 4-octyl itaconate protects human umbilical vein endothelial cells from high glucose. Biochem. Biophys. Res. Commun. 508, 921-927 (2019).

17. Tang, C. et al. 4-Octyl itaconate activates Nrf2 signaling to inhibit proinflammatory cytokine production in peripheral blood mononuclear cells of systemic lupus erythematosus patients. Cell Physiol. Biochem. 51, 979-990 (2018).

18. Liu, H. et al. Four-octyl itaconate activates Keap1-Nrf2 signaling to protect neuronal cells from hydrogen peroxide. Cell Commun. Signal. 16, 81 (2018).

19. Ding, $H$. et al. Dexamethasone-induced apoptosis of osteocytic and osteoblastic cells is mediated by TAK1 activation. Biochem. Biophys. Res. Commun. 460, 157-163 (2015).

20. $\mathrm{Xu}, \mathrm{Y}$. et al. Hepcidin increases intracellular $\mathrm{Ca}^{2+}$ of osteoblast hFOB1.19 through L-type Ca ${ }^{2+}$ channels. Regul. Pept. 172, 58-61 (2011).

21. Xue, J. et al. Lnc-THOR silencing inhibits human glioma cell survival by activating MAGEA6-AMPK signaling. Cell Death Dis. 10, 866 (2019).

22. Xu, X. Z. et al. Targeting Keap1 by miR-626 protects retinal pigment epithelium cells from oxidative injury by activating Nrf2 signaling. Free Radic. Biol. Med. 143, 387-396 (2019).
23. Zhang, $H$. et al. Salvianolic acid A protects RPE cells against oxidative stress through activation of Nrf2/HO-1 signaling. Free Radic. Biol. Med. 69, 219-228 (2014).

24. Liu, H. et al. K6PC-5 activates SphK1-Nrf2 signaling to protect neuronal cells from oxygen glucose deprivation/re-oxygenation. Cell Physiol. Biochem. 51, 1908-1920 (2018).

25. Li, K. R. et al. Ginsenoside Rg-1 protects retinal pigment epithelium (RPE) cells from cobalt chloride $(\mathrm{CoCl} 2)$ and hypoxia assaults. PLOS ONE 8, e84171 (2013).

26. Zhang, Y. M. et al. Requirement of Galphai1/3-Gab1 signaling complex for keratinocyte growth factor-induced PI3K-AKT-mTORC1 activation. J. Investig. Dermatol. 135, 181-191 (2015).

27. Brooks, M. M., Neelam, S., Fudala, R., Gryczynski, I. \& Cammarata, P. R. Lenticular mitoprotection. Part A: monitoring mitochondrial depolarization with JC-1 and artifactual fluorescence by the glycogen synthase kinase-3beta inhibitor, SB216763. Mol. Vis. 19, 1406-1412 (2013).

28. Zitka, O. et al. Redox status expressed as GSH:GSSG ratio as a marker for oxidative stress in paediatric tumour patients. Oncol. Lett. 4, 1247-1253 (2012).

29. Zhao, S. et al. MicroRNA-200a activates Nrf2 signaling to protect osteoblasts from dexamethasone. Oncotarget 8, 104867-104876 (2017).

30. She, C., Zhu, L. Q., Zhen, Y. F., Wang, X. D. \& Dong, Q. R. Activation of AMPK protects against hydrogen peroxide-induced osteoblast apoptosis through autophagy induction and NADPH maintenance: new implications for osteonecrosis treatment? Cell Signal. 26, 1-8 (2014).

31. Park, B. G., Yoo, C. I., Kim, H. T., Kwon, C. H. \& Kim, Y. K. Role of mitogenactivated protein kinases in hydrogen peroxide-induced cell death in osteoblastic cells. Toxicology 215, 115-125 (2005).

32. Sung, H. K., Song, E., Jahng, J. W. S., Pantopoulos, K. \& Sweeney, G. Iron induces insulin resistance in cardiomyocytes via regulation of oxidative stress. Sci. Rep. 9, 4668 (2019).

33. Celeghini, E. C. C. et al. Efficiency of CellROX deep red((R)) and CellROX orange $((R))$ fluorescent probes in identifying reactive oxygen species in sperm samples from high and low fertility bulls. Animal Biotechnol. 1-7 (2019).

34. Yang, L. et al. Stem cell factor (SCF) protects osteoblasts from oxidative stress through activating c-Kit-Akt signaling. Biochem. Biophys. Res. Commun. 455, 256-261 (2014).

35. Zhang, X. Y., Shan, H. J., Zhang, P., She, C. \& Zhou, X. Z. LncRNA EPIC1 protects human osteoblasts from dexamethasone-induced cell death. Biochem. Biophys. Res. Commun. 503, 2255-2262 (2018).

36. Halestrap, A. P. Calcium, mitochondria and reperfusion injury: a pore way to die. Biochem. Soc. Trans. 34, 232-237 (2006).

37. Halestrap, A. Biochemistry: a pore way to die. Nature 434, 578-579 (2005).

38. Halestrap, A. P., Gillespie, J. P., O'Toole, A. \& Doran, E. Mitochondria and cell death: a pore way to die? Symp. Soc. Exp. Biol. 52, 65-80 (2000)

39. Pajares, M., Cuadrado, A. \& Rojo, A. I. Modulation of proteostasis by transcription factor NRF2 and impact in neurodegenerative diseases. Redox Biol. 11, 543-553 (2017).

40. Yang, Y. et al. An overview of the molecular mechanisms and novel roles of Nrf2 in neurodegenerative disorders. Cytokine Growth Factor Rev. 26, 47-57 (2015).

41. Xiong, W., MacColl Garfinkel, A. E., Li, Y., Benowitz, L. I. \& Cepko, C. L. NRF2 promotes neuronal survival in neurodegeneration and acute nerve damage. J. Clin. Investig. 125, 1433-1445 (2015).

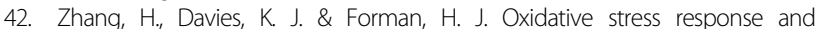
Nrf2 signaling in aging. Free Radic. Biol. Med. 88, 314-336 (2015).

43. Schafer, M. \& Werner, S. Nrf2-a regulator of keratinocyte redox signaling. Free Radic. Biol. Med. 88, 243-252 (2015).

44. Baines, C. P. et al. Loss of cyclophilin D reveals a critical role for mitochondrial permeability transition in cell death. Nature 434, 658-662 (2005). 